\title{
Effects of bone marrow-derived stem cells on necrotic and apoptotic cell death induced by renal ischemia- reperfusion in rats
}

\author{
Yang Wang ${ }^{1}$, Zhi Jian Wang ${ }^{1}$, Gong Xian Wang ${ }^{1}$, An Xie $^{1}$ \\ ${ }^{1}$ Institute of Urology, First Affiliated Hospital, Nanchang University, Nanchang 330006, China
}

Ischemia-reperfusion injury (IRI) is a crucial element in the pathogenesis of acute ischemic renal failure and can importantly influence the early functional recovery and even long-term survival of a transplanted kidney. It is generally thought that renal repair following IRI requires the replacement or regeneration of renal tubular epithelial cells that are detached or damaged. Recently, it has been reported that intravenously infused adult bone marrow-derived stem cells (BMSCs) are able to migrate to injured kidney tissue and differentiate into renal cells, potentially ameliorating the impairment of renal function. Nevertheless, the mechanisms by which bone marrowderived stem cells promote the repair of injured kidney tissue have not been completely elucidated by animal studies. Bone marrow mononuclear cells(BMMNCs) transplantation has been reported to efficiently improve ischemic heart disease. BMMNCs may contain mesenchymal stem cells and hemopoietic stem cells or endothelial progenitor cells as well as other types of cells, such as macrophages, lymphocytes and fibroblasts, which may afford beneficial microenvironments for stem cells. In the present study, we investigated the distribution and contribution of transplanted BMMNCs in the IRI rat kidney. Furthermore, the effect of BMMNCs on IR-induced necrotic or apoptotic death of rat renal tubular cells was observed and analyzed, so as to investigate whether bone marrowderived stem cell transplantation aids in renal tubule repair and helps maintain the structural integrity of renal tubules following IRI. Results demonstrated that many transplanted BMMNCs (labeled with DAPI) were found in the injured kidney, and some of these cells were located in the renal tubular epithelium Results also indicated that the transplantation of BMMNCs significantly reduced IR-induced necrosis, degeneration and apoptosis of renal tubular epithelial cells, and promoted renal tubular epithelial cell proliferation following IRI. Together, these findings suggest that the transplantation of BMMNCs can help restore and maintain the structural integrity of renal tubules after IRI. Not only does this provide insights into the mechanism of renal tissue repair, but it also provides a basis for development of new strategies for treatment of the renal lesion.

Keywords: bone marrow mononuclear cells, apoptosis, kidney, necrosis, renal ischaemic injury

Cell Research (2008) 18:s80. doi: 10.1038/cr.2008.170; published online 4 August 2008

Correspondence: Yang Wang

E-mail: wangy63cn@sina.com 